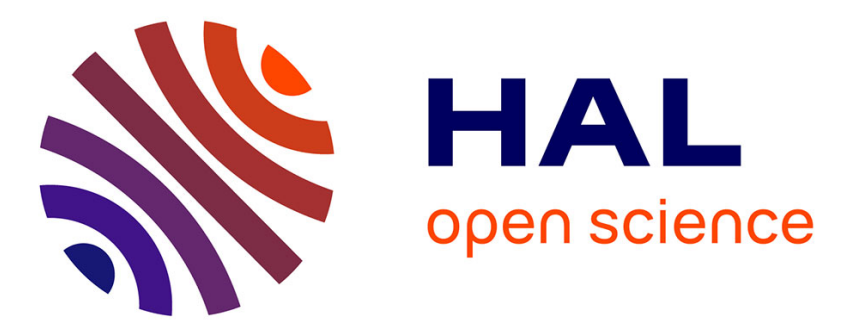

\title{
Experimental generation of spherical converging shock waves
}

\author{
Mathieu Brasseur, Marc Vandenboomgaerde, Christian Mariani, Diogo C \\ Barros, Denis Souffland, Georges Jourdan
}

\section{- To cite this version:}

Mathieu Brasseur, Marc Vandenboomgaerde, Christian Mariani, Diogo C Barros, Denis Souffland, et al.. Experimental generation of spherical converging shock waves. Experiments in Fluids, 2021, 62 (7), 10.1007/s00348-021-03248-x . hal-03283892

\section{HAL Id: hal-03283892 \\ https://hal-amu.archives-ouvertes.fr/hal-03283892}

Submitted on 12 Jul 2021

HAL is a multi-disciplinary open access archive for the deposit and dissemination of scientific research documents, whether they are published or not. The documents may come from teaching and research institutions in France or abroad, or from public or private research centers.
L'archive ouverte pluridisciplinaire HAL, est destinée au dépôt et à la diffusion de documents scientifiques de niveau recherche, publiés ou non, émanant des établissements d'enseignement et de recherche français ou étrangers, des laboratoires publics ou privés. 


\title{
Experimental generation of spherical converging shock waves
}

\author{
Mathieu Brasseur - Marc Vandenboomgaerde - Christian Mariani \\ Diogo C. Barros · Denis Souffland · Georges Jourdan
}

Received: date / Accepted: date

\begin{abstract}
Spherical converging shock waves with incoming Mach number 1.18 are generated in a conical test chamber fitted to a conventional square-section shock tube. A wave cutter is employed to ensure a proper shock transition from the square to a cylindrical straight pipe installed upstream of the convergent section. The incident planar wave is transformed into a spherical shock by the use of an ellipsoidal membrane acting as a gas lens separating two gases with different densities. The transmitted shock wave propagates within the conical section, and accelerates towards the apex. The trajectory and the shape of the shock wave are both characterized by planar Mie scattering. The spherical shock follows the similarity solution proposed by Guderley (1942), whose trajectory agrees very well with the numerical simulation of the Chester-Chisnell-Whitham (CCW) theory for the geometry considered. The procedure demonstrated here provides a potential method for future investigations of shock focusing and Richtmyer-Meshkov instability in spherical geometry.
\end{abstract}

Keywords Shock wave focusing $\cdot$ Flow visualization

\section{Introduction}

Shock waves have been widely investigated in natural and engineering sciences. In particular, they can be encountered in supernova collapse, inertial confinement fusion process and detonation-driven shocks (Apazidis and Eliasson, 2018). In all these applications, the convergence of the shock wave, i.e. shock focusing, is of primary importance.

M. Brasseur, C. Mariani, D.C. Barros and G. Jourdan

Aix Marseille Université, CNRS, IUSTI, Marseille, France

E-mail: mathieu.brasseur@univ-amu.fr

M. Vandenboomgaerde and D. Souffland

CEA-DAM, DIF, 91297 Arpajon, France
In his seminal work, Guderley (1942) proposed a similarity solution for the propagation of converging shock waves where the shock wave radius follows a power law in time $-t^{\alpha}$ (measured backwards with respect to the time when the shock arrives at the converging center) and $\alpha$ is the similarity exponent. The working gas and the cylindrical or spherical nature of the shock focusing phenomena set the value of $\alpha$. Later, Chester (1954), Chisnell (1957) and Whitham $(1958,1974)$ provided a general framework to approximate the propagation of shock waves through varying section channels with finite area changes, known as the CCW governing equations. Their analysis demonstrated a good agreement with the similarity solutions proposed by Guderley (1942). Further numerical investigations conducted by Lazarus and Richtmyer (1977), Hafner (1988) and Ramsey et al (2012) characterized the similarity exponent for multiple gases and conditions, providing very accurate values of $\alpha$. The analytical results of Chisnell (1998) confirmed the previous numerical computations of the CCW equations.

Shock focusing has been the subject of multiple experimental investigations, particularly in cylindrical geometries. Perry and Kantrowitz (1951) generated converging shock waves in a "teardrop", axially symmetric annular shock tube test section, and noted the presence of high temperature spots at the converging center of a moderate strength shock wave. The stability of cylindrical shock waves was investigated by Takayama et al (1987), where two annular shock tubes were considered. Although the cylindrical converging shock waves were unstable and sensitive to the structure of each facility, their trajectories exhibited a compelling match with the similarity solution of Guderley (1942) for Mach numbers ranging from 1.1 to 2.1. More recently, Zhai et al (2010) generated perfectly cylindrical shock waves at Mach number 1.2 , within a window of $15^{\circ}$. They employed converging curved walls designed using the shock dynamics equations. Following the gas lens framework proposed by $\mathrm{Di}-$ 
motakis and Samtaney (2006) and generalized by Vandenboomgaerde and Aymard (2011), Biamino et al (2014) generated cylindrical shock waves within a converging channel issued from a square-section shock tube. A well defined 3Dprinted grid overlaid by a nitrocellulose membrane was used to separate two gases, creating the interface required to bend the shock. Both techniques have been employed in the past few years for the study of the Richtmyer-Meshkov instability in cylindrical configurations (Biamino et al, 2015; Vandenboomgaerde et al, 2018; Luo et al, 2018, 2019).

Other investigations were devoted to the experimental generation of spherical converging shock waves. A conical converging channel was used by Russell (1967) to connect two shock tube sections, leading to a significant shock wave acceleration matching the predictions of the CCW model. Setchell et al (1972) investigated a conical section in the range of Mach numbers from 6 to 10 operating a shock velocity probe installed inside the section. Thanks to a transparent spherical chamber, Hosseini and Takayama (2005) studied the convergence of spherical shock waves using optical visualization. Micro-explosives were detonated at the center of the chamber shaped according to optical refraction laws, allowing shock visualization. Kjellander et al (2012) generated spherical converging shock waves employing parameterized curved walls in a circular cross-section shock tube. By focusing the shock wave, they managed to produce light pulses and reached apparent temperatures up to 27 $000 \mathrm{~K}$. This technique was employed later to study convergence effects on temperature (Liverts and Apazidis, 2016) or to generate strong pressure jumps in conventional shock tubes (Sembian and Liverts, 2020).

In most of the experiments described above, little was reported about the geometry of the propagating shock wave, partially due to the inherent difficulties arising from the optical visualization in 3D configurations. Here, we demonstrate the generation of a spherical converging shock wave using an experimental gas lens that separates air and sulfur hexafluoride $\left(\mathrm{SF}_{6}\right)$. Shock focusing is directly visualized by means of a transparent conical chamber, illuminated with a laser sheet that provides information about the shock wave geometry and the trajectory through planar Mie scattering.

\section{Experimental setup}

\subsection{Shock tube and convergent test section}

The experiments were performed using the square-section shock tube $\left(80 \times 80 \mathrm{~mm}^{2}\right)$ described in Jourdan et al (2004). Figure 1 displays a sketch of the experimental setup. To generate the planar shock wave propagating through air, we used a diaphragm composed of three superposed aluminium sheets of $12 \mu \mathrm{m}$ thicknesses. The diaphragm separates the driver and driven sections of the shock tube. In order to characterize the shock wave propagation after the diaphragm burst, the driven section of the shock tube is equipped with two flush-mounted pressure sensors (PCB 113A26 with response time less than $1 \mu \mathrm{s}$ ) connected to a PCB 482C signal conditioner. We acquired the pressure jump signals using a multichannel oscilloscope (Teledyne Lecroy Wavesurfer 3054).

A wave cutter was placed at the end of the shock tube driven section (see figure 1(a)). This straight cylinder of length $165 \mathrm{~mm}$ ensures a proper shock transition from the square cross-section chamber to the circular one. Further downstream, we installed a $150 \mathrm{~mm}$ transparent PMMA cylindrical chamber equipped with two other pressure sensors. Preliminary tests were conducted to verify that the length of the straight cylinder is sufficient to ensure a proper transition. The velocity was determined from pressure sensors $C_{1}$ and $C_{2}$ for the square section, $C_{3}$ and $C_{4}$ for the axisymmetric one, as shown in figure 1(a). For a range of incident Mach numbers between 1.1 and 1.5 , the shock velocity variation was less than $2 \%$ when the shock moved from the square to the axisymmetric section. Figure 2 shows the quality of flatness of the resulting planar shock wave.

The generation of a converging shock wave using the gas lens technique requires the use of an interface that separates the straight and the converging sections, each filled with two different gases. To do so, we used a $0.9 \mu \mathrm{m}$-thick polyester mylar film superimposed onto a 3D-printed grid. Following Dimotakis and Samtaney (2006) and Vandenboomgaerde and Aymard (2011), we define the shape of the interface as a surface cut of the ellipsoid of revolution described by its elliptical cross-section:

$r(\theta)=r_{o} \frac{1-e}{1-e \cos (\theta)}$,

with :

$r\left(\theta_{0}\right)=\frac{h}{\sin \left(\theta_{0}\right)}$.

Here, $e$ is the ellipse eccentricity, $h$ is the shock tube radius, and $(r, \theta)$ are the associated polar coordinates. In this coordinate system, the pole $O$ is also a focus of the ellipse, and $x$ is the major axis. $r_{o}$ represents the distance between the pole and the point of the ellipse on the major axis. The eccentricity reads $e=W_{t} / W_{i}$, where $W_{i}$ and $W_{t}$ are the incident and the transmitted shock velocities, respectively (Vandenboomgaerde and Aymard, 2011). The pair of gases considered is air and $\mathrm{SF}_{6}$. Details of the principle of the gas lens and the associated geometrical parameters are given in figure 3 .

We designed the gas lens for an optimal Mach number of the incident shock wave in air, $M_{i}$, which is equal to 1.15 . This leads to $W_{i}=394 \mathrm{~ms}^{-1}$, and $W_{t}=165 \mathrm{~ms}^{-1}$. The resulting eccentricity is $e=0.42$. As $h=40 \mathrm{~mm}$, and the half 
(a)

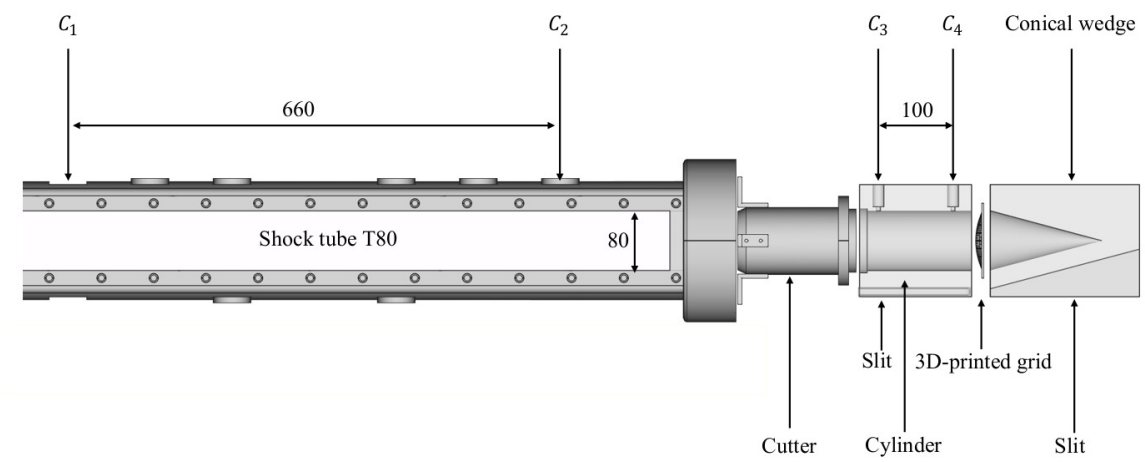

(b)

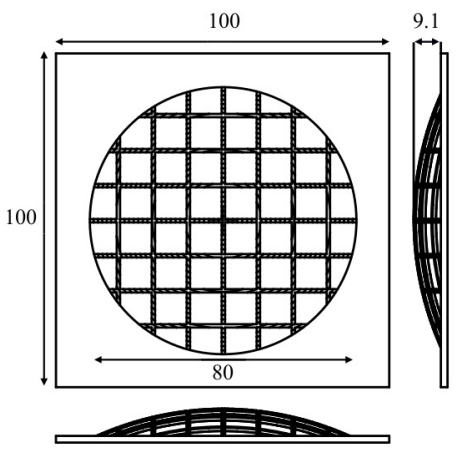

Fig. 1 Experimental setup: (a) Side view depicting the square-section shock tube, the axisymmetric wave cutter, the intermediate cylinder, the grid and the conical test section. The locations of the four pressure sensors are specified in the sketch. (b) Geometry of the 3D-printed grid used to separate the straight cylinder and the conical channel. All dimensions are in millimeters (mm).
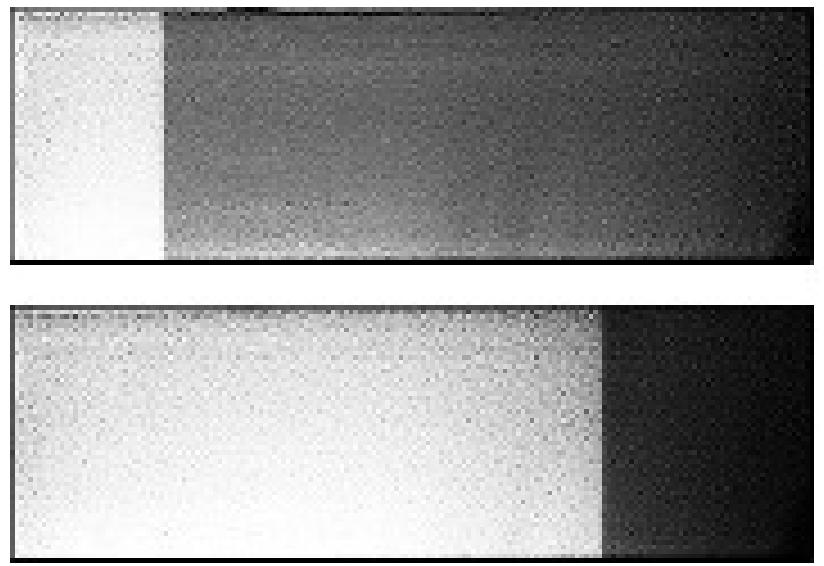

Fig. 2 Visualization of the normal shock wave travelling from left to right in the cylindrical chamber. Details of the imaging parameters are described in section 2.2.

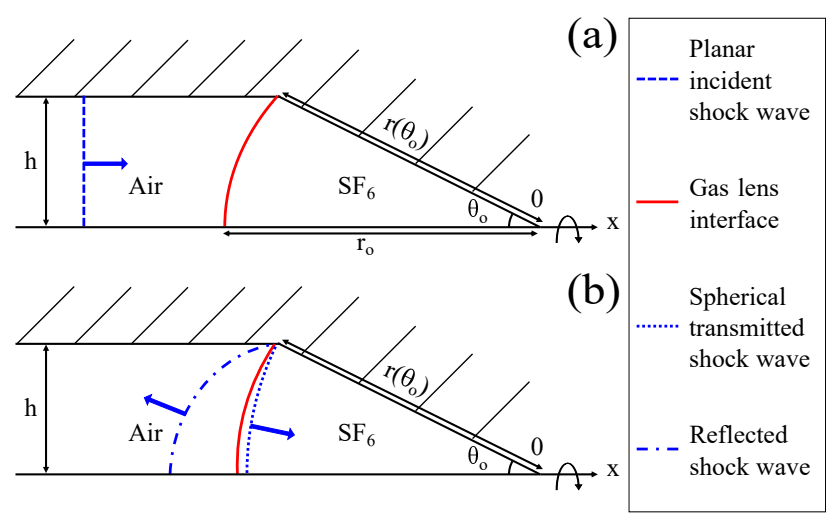

Fig. 3 Sketch of the gas lens configuration with the geometrical parameters : (a) before shock-interface interaction (b) after interaction.

angle at the cone apex is $\theta_{0}=15^{\circ}$, the radius of the transmitted shock wave immediately after passing through the gas lens is $r\left(\theta_{0}\right)=155 \mathrm{~mm}$. Finally, the transmitted shock wave propagates into a PMMA conical chamber with length
$149 \mathrm{~mm}$ from the base to the apex. Due to the manufacturing precision near the apex, the exact conical chamber length is $L=146 \mathrm{~mm}$. Figure 1(b) illustrates the grid that materializes the lens. It is discretized in a uniform square mesh $(9.5 \times 9.5$ $\mathrm{mm}^{2}$ ) with $1 \mathrm{~mm}$-thick rods, and was painted in black to reduce reflection. A slit of thickness $10 \mathrm{~mm}$ was cut at the bottom of the acrylic bloc encasing the conical chamber to facilitate its illumination. It is of interest to note that the gas lens design is robust to $M_{i}$ uncertainties. For example, if the $e=0.42$ lens were used for $M_{i}=1.2$, the transmitted wave would display a $0.1 \mathrm{~mm}$ radius deviation from the expected $155 \mathrm{~mm}$ spherical shock wave for $M_{i}=1.15$.

\subsection{Visualization technique}

The three-dimensional geometry of both the cylindrical and the convergent test sections precludes the use of common schlieren or shadowgraph techniques to visualize the shock wave propagation. Here, we apply a planar Mie scattering technique to visualize the shock wave motion (Clemens and Mungal, 1991; Tropea, 2011). The convergent chamber filled with $\mathrm{SF}_{6}$ was seeded with oil droplets with diameter between 1 and $3 \mu \mathrm{m}$ using an atomizer (Moutte, 2018). For these micron-sized droplets, the estimated response time is about 3 to $30 \mu \mathrm{s}$. They are therefore suitable for the current set of experiments (Williams et al, 2015). We employed a 532 nm continuous RayPower 5000 laser (Dantec Dynamics) to generate a $1 \mathrm{~mm}$-thick laser sheet. The laser sheet goes through the slit in the acrylic bloc and illuminates the vertical symmetry plane of the conical chamber. The laser was operated at the maximum power of $5 \mathrm{~W}$.

The passage of the shock wave through the seeded volume induces a local change of density, increasing the light scattering as illustrated in figure 2. The same method was applied in the conical chamber. The images were acquired 
by an ultra high-speed Phantom v2512 camera (Ametek, 12 bit with a sensor pixel size of $\Delta_{\text {pix }}=28 \mu \mathrm{m}$ ) operated at a sampling rate $1 / \Delta t$ of $70 \mathrm{kHz}$ and a resolution of $640 \times 480$ pixels. The camera was fitted with a Nikon AF-S Nikkor $50 \mathrm{~mm}$ lens with a numerical aperture of $f_{\#}=1.4$. The resulting magnification was 0.11 . To avoid image blurring due to the shock wave motion, the exposure time was set to $680 \mathrm{~ns}$.

The conical geometry of the test chamber distorts the scales in the laser sheet plane. In order to reconstruct the undistorted picture, an image processing algorithm needs to be elaborated. Thus, a calibration grid consisting of a $5 \mathrm{~mm}-$ square pattern was placed in the symmetry plane of the convergent chamber, as depicted in figure 4(a). Figure 4(b) shows the undistorted grid image obtained after the removal of the chamber. A map between both data sets was obtained using the regression learner application of the machine learning toolbox in Matlab (Mathworks, Inc.). To generate the map between both data sets, we manually selected the intersections between black and white squares in both undistorted and distorted pictures. For the current geometry, the distortion depends on both axes. We trained the algorithms to find the undistorted data set from the distorted one. After training all the algorithms in the toolbox, we selected the one with the lowest root mean square error between effective and predicted data sets. The best algorithm following this criterion is the Matérn 5/2 Covariance function. It is a Gaussian Process Regression model. The corrected image (see figure 4(c)) and original grid points from figure 4(b) presented a root mean square error within sub pixel accuracy of 0.8 pixel, corresponding to approximately $0.2 \mathrm{~mm}$.

\section{Results and discussion}

We present here three representative runs. For these runs, the incident shock wave travels in air at atmospheric pressure $(101.3 \mathrm{kPa})$ and ambient temperature $(295.15 \mathrm{~K})$ with a Mach number $M_{i}=1.18$. We used the pressure sensors (see figure 1) to determine the shock velocity. The incident shock wave is transmitted into $\mathrm{SF}_{6}$ at the same thermodynamic conditions. The theoretical velocity of the transmitted shock wave is $172 \mathrm{~ms}^{-1}$. However, the measured velocity is only $W_{t}=164 \mathrm{~ms}^{-1}$. This reduced shock strength is due to the energy spent on the material interface breaking. The theoretical perturbation of the sphericity due to this weaker transmitted shock wave $\left(M_{t}=1.21\right)$ is negligible, as presented later is section 3.1. Figure 5 presents both raw and processed images of the transmitted shock wave at different times for one of the runs. Let us define $R(\theta, t)$ as the polar coordinate of the shock wave location. The zero time is defined as the moment when the transmitted shock wave is fully visible into the conical module $(R(\theta, t=0)=L)$.

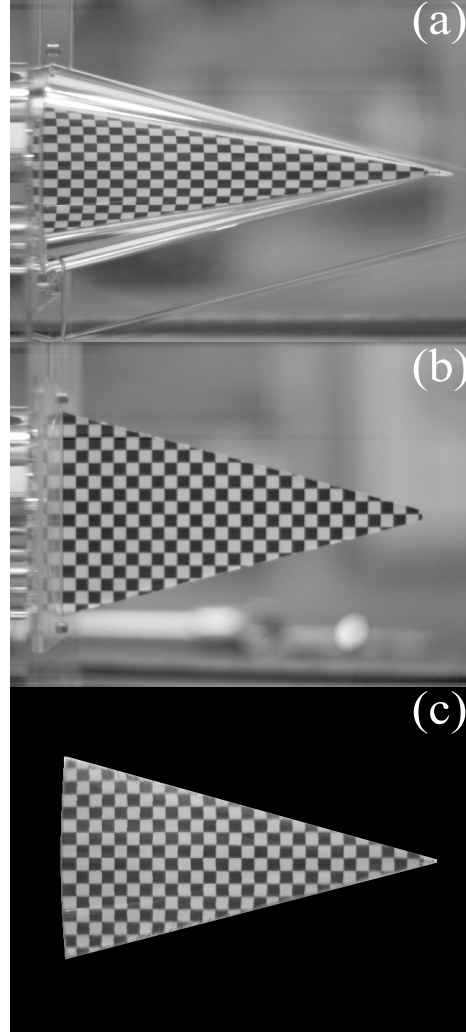

Fig. 4 Image processing: (a) raw grid image inside the conical chamber (b) reference grid (c) processed version of (a) obtained from the calibration model.

The images depict the spherical transmitted shock wave which moves from left to right in the conical test chamber. This spherical shock can be clearly visualized through its whole transit along the cone. This demonstrates the good reliability of the visualisation method. We note the influence of the gas-lens supporting grid on the flow behind the shock, with the development of the perturbations at the air/SF $\mathrm{S}_{6}$ interface, specific of the behavior of a Richtmyer-Meshkov instability. However, the transmitted shock remains stable and its shape shows no influence from the grid, the membrane remnants or the instability.

\subsection{Shock wave sphericity}

In order to determine the shape $R(\theta, t)$ of the shock front, the images were post-processed. Far from the apex, the low and high average signal intensities across the spherical shock were 279 and 341, respectively. Near the apex, they were about 231 and 566. This means that light-scattering increased with shock focusing. For these experiments, we estimated a noise intensity of 17 units. The contrast of the images was adjusted, and they were smoothed using a Gaussian filter. The resulting image was sharpened using an unsharp mask before being binarized. The threshold was adjusted man- 

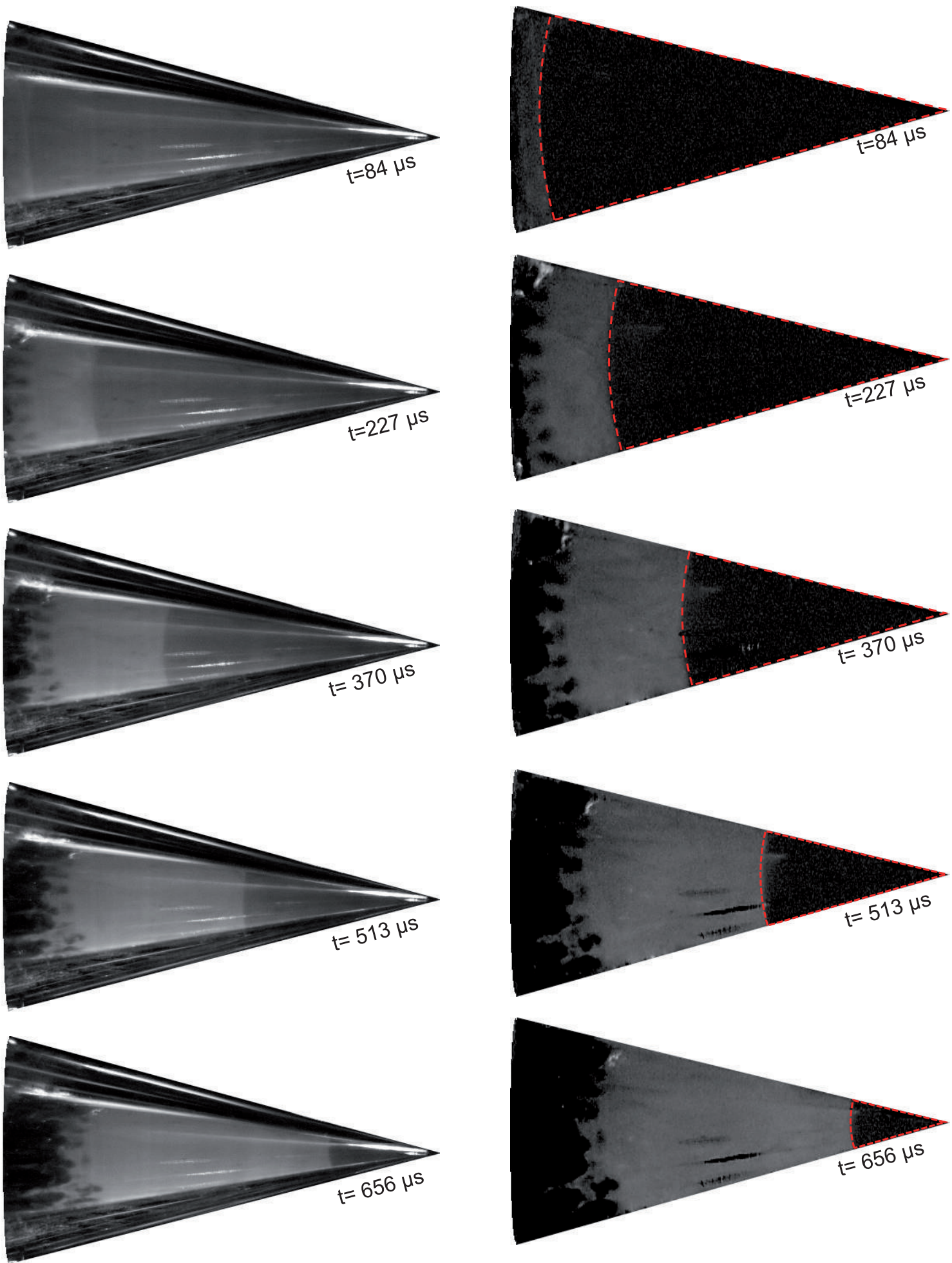

Fig. 5 Snapshots of raw (left) and processed (right) images showing the propagation of a spherical converging shock wave issued from the refraction of a planar shock wave $\left(\mathrm{M}_{i}=1.18\right.$ in air) through an air/ $\mathrm{SF}_{6}$ ellipsoidal interface. The shock moves from the left to the right. The dashed lines represent the circular radius obtained from the image processing described in section 2.2 . 

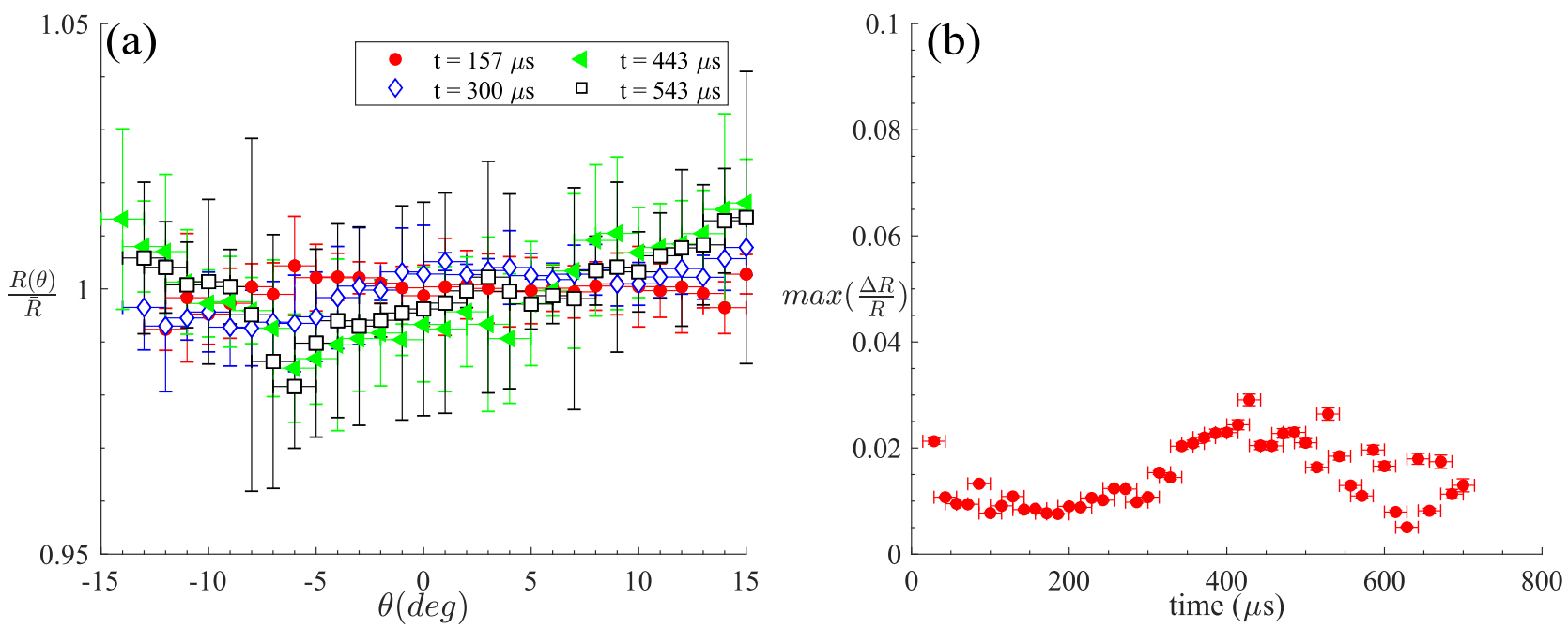

Fig. 6 Characterization of the shock front curvature and sphericity. a) Radial deviation $R(\theta, t) / \bar{R}(t)$ of the shock front for four snapshots. b) Maximum deviation $\max (\Delta R / \bar{R})$ of the shock front over time for one experimental run.

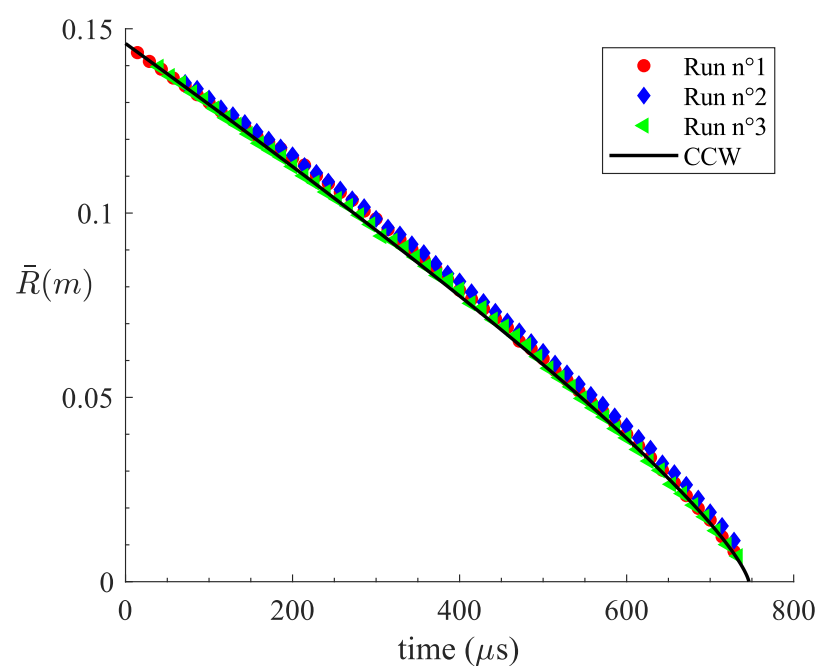

Fig. 7 Shock wave trajectories. Symbols and continuous line represent the three experimental runs, and the numerical solution of equation 2 initialized with $M_{t}=1.21$, respectively. The estimated error of the radius is $\pm 3 \mathrm{~mm}$.

ually, as the light scattering increased during shock convergence. We obtained a black (unshocked area) and white (shocked area) image. The shape of the shocked medium is extracted using a standard edge detector. Finally, the line corresponding to the shock front is selected. Following Kjellander et al (2012), we qualify the shock wave sphericity using two criteria. The first criterion is the radial deviation defined as $R(\theta, t) / \bar{R}(t)$, where $\bar{R}(t)$ is the mean radius of the shock wave. The second criterion is the maximum of the relative deviation, $\max (\Delta R / \bar{R})=\max ((R(\theta, t)-\bar{R}(t)) / \bar{R}(t))$, with $\Delta R$ in absolute value. Figure 6(a) shows the radial deviation for four different times. It remains smaller than $4 \%$,

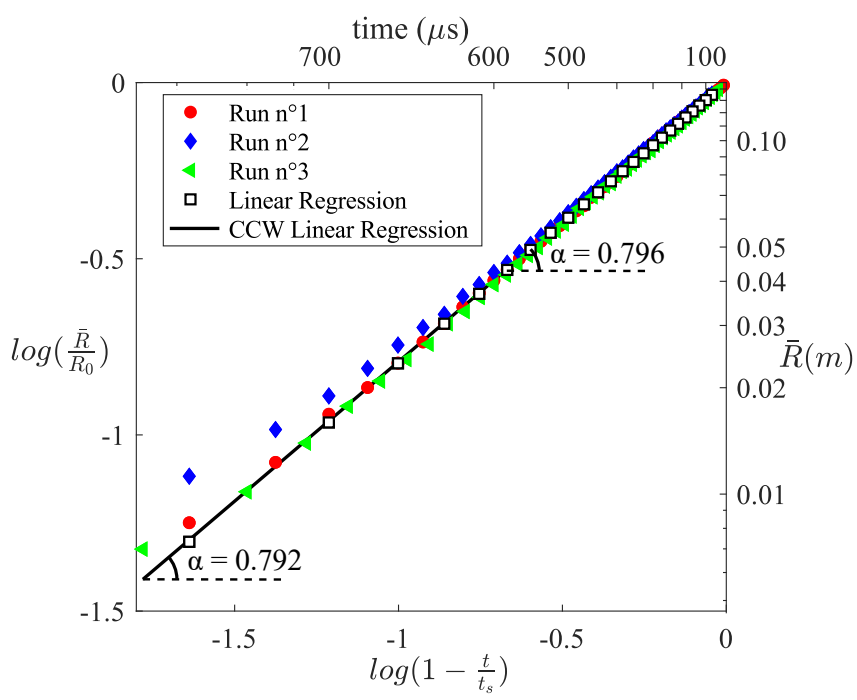

Fig. 8 Dimensionless trajectories of the shock wave in the cone. White squares corresponds to a linear regression of the three runs under consideration.

which means that the real shape of the shock front is close to a perfect sphere. This is confirmed by the maximum relative deviation which also remains smaller than $4 \%$ (see figure 6(b)).

\subsection{Shock wave trajectory}

The trajectory of the spherical converging shock wave is also considered. Experimental trajectories are compared to a numerical solution of the CCW approximate geometrical theory (Chester, 1954; Chisnell, 1957; Whitham, 1958) : 
$\frac{M_{t}}{M_{t}^{2}-1} \lambda\left(M_{t}\right) \frac{d M_{t}}{d r}+\frac{1}{A} \frac{d A}{d r}=0$,

with :

$\lambda\left(M_{t}\right)=\left(1+\frac{2}{\gamma+1} \frac{1-\mu^{2}}{\mu}\right)\left(1+2 \mu+\frac{1}{M_{t}^{2}}\right)$,

$\mu^{2}=\frac{(\gamma-1) M_{t}^{2}+2}{2 \gamma M_{t}^{2}-(\gamma-1)}$.

where $M_{t}$ and $A$ are the Mach number and the area of the transmitted shock wave in $\mathrm{SF}_{6}$, respectively. The area $A$ is proportional to $r^{2}$ in the spherical case. $\mu$ stands for the Mach number (in the shock reference frame) behind the transmitted shock wave. As $M_{t}=(d r / d t) / C_{s}$ where $C_{s}$ is the sound speed in $\mathrm{SF}_{6}$, we obtained the evolution of the shock position as a function of time using numerical integration with the software Mathematica (Wolfram Research, Inc.).

In the strong shock limit $\left(M_{t} \rightarrow \infty\right)$, this theory is in good agreement with the similarity solution obtained by Guderley (1942) :

$$
\frac{R}{R_{0}}=\left(1-\frac{t}{t_{s}}\right)^{\alpha}
$$

where $R_{0}$ is the initial shock radius, and $t_{s}$ the shock collapsing time. For a strong shock, $\alpha$ is a function of the geometry (spherical or cylindrical) and of the adiabatic exponent $\gamma$.

Figure 7 presents the measured trajectories for three different runs under consideration. The experimental trajectory is in good agreement with the numerical integration of the $\mathrm{CCW}$ theory. In figure 8 , we present the dimensionless trajectories based on the scaling of equation 4 , and the corresponding similarity exponents, which are compared with previous investigations in table 1 . In our experiments, the estimation of the shock collapsing time $t_{s}$ is crucial in the determination of $\alpha$, and the accuracy of this estimation is greatly limited by the camera's sampling rate and the image resolution near the apex. Decreasing the shock collapsing time by one snapshot $\left(t_{s} \rightarrow t_{s}-\Delta t\right)$ resulted in a variation of -0.045 for $\alpha$. On the other hand, if we overestimated $t_{s}$ by one snapshot $\left(t_{s} \rightarrow t_{s}+\Delta t\right), \alpha$ varied by +0.021 . The resulting estimated uncertainty of the experimental similarity exponent is 0.033 , corresponding to less than $5 \%$ of $\alpha$. Although the similarity exponent compares very well to the values of $\alpha$ reported by Lazarus (1981); Hafner (1988); Chisnell (1998); Ramsey et al (2012), it is important to note that their investigations considered the motion of strong shock waves, which does not include our initial transmitted Mach number $M_{t}=1.21$. However, as the shock wave accelerates towards the apex, its strength increases, and the flow better approximates a self-similar behavior.
Table 1 Self-similarity exponent of spherical converging shock waves.

\begin{tabular}{lll}
\hline Work & $\gamma$ & value of $\alpha$ \\
\hline Experimental fit & 1.09 & $0.796 \pm 0.033$ \\
CCW Method fit & 1.09 & 0.792 \\
Lazarus (1981) & 1.07 & 0.815 \\
& 1.10 & 0.796 \\
Hafner (1988) & 1.10 & 0.796 \\
Chisnell (1998) & 1.09 & 0.802 \\
Ramsey et al (2012) & 1.10 & 0.775 \\
\hline
\end{tabular}

\section{Conclusions}

A conventional shock tube has been adapted to generate spherical converging shock waves in a conical test section. The planar incident shock was transformed into a spherical wave by using an experimental gas lens following the theory proposed by Dimotakis and Samtaney (2006). Planar Mie scattering was employed to visualize the shock front and its trajectory through the convergent section. The geometrical distortion of the wedge was corrected by means of a specific image calibration procedure.

The results demonstrated the generation of an almost perfect spherical shock front throughout convergence. Moreover, the wave trajectory agrees very well with the CCW shock theory and the similarity solution proposed by Guderley (1942). The similarity exponent was remarkably similar to previous theoretical and numerical computations in spherical geometry. The methodology described here provides a potential framework to create repeatable initial conditions for shock focusing in 3D configurations, for example in the study of converging Richtmyer-Meshkov instability extending previous cylindrical configurations (Biamino et al, 2015; Vandenboomgaerde et al, 2018).

Acknowledgements This work is supported by CEA/DAM/DIF under contract \#BACO/AJ-20-73/C37653. The authors sincerely thank Lazhar Houas for his careful reading of the manuscript. This is a postpeer-review, pre-copyedit version of an article published in Experiments In Fluids. The final authenticated version is available online at: http://dx.doi.org/10.1007/s00348-021-03248-x.

\section{References}

Apazidis N, Eliasson V (2018) Shock focusing phenomena. Springer

Biamino L, Mariani C, Jourdan G, Houas L, Vandenboomgaerde M, Souffland D (2014) Planar shock focusing through perfect gas lens: first experimental demonstration. Journal of Fluids Engineering 136(9)

Biamino L, Jourdan G, Mariani C, Houas L, Vandenboomgaerde M, Souffland D (2015) On the possibility of studying the converging Richtmyer-Meshkov instability in a conventional shock tube. Experiments in Fluids 56(2):26 
Chester W (1954) The quasi-cylindrical shock tube. The London, Edinburgh, and Dublin Philosophical Magazine and Journal of Science 45(371):1293-1301

Chisnell R (1957) The motion of a shock wave in a channel, with applications to cylindrical and spherical shock waves. Journal of Fluid Mechanics 2(3):286-298

Chisnell R (1998) An analytic description of converging shock waves. Journal of Fluid Mechanics 354:357-375

Clemens N, Mungal M (1991) A planar Mie scattering technique for visualizing supersonic mixing flows. Experiments in Fluids 11(2-3):175-185

Dimotakis P, Samtaney R (2006) Planar shock cylindrical focusing by a perfect-gas lens. Physics of Fluids 18(3):031,705

Guderley K (1942) Starke kugelige und zylindrische verdichtungsstosse in der nahe des kugelmitterpunktes bnw. der zylinderachse. Luftfahrtforschung 19:302

Hafner P (1988) Strong convergent shock waves near the center of convergence: A power series solution. SIAM Journal on Applied Mathematics 48(6):1244-1261

Hosseini S, Takayama K (2005) Implosion of a spherical shock wave reflected from a spherical wall. Journal of Fluid Mechanics 530:223

Jourdan G, Houas L, Schwaederlé L, Layes G, Carrey R, Diaz F (2004) A new variable inclination shock tube for multiple investigations. Shock Waves 13(6):501-504

Kjellander M, Tillmark N, Apazidis N (2012) Energy concentration by spherical converging shocks generated in a shock tube. Physics of Fluids 24(12):126,103

Lazarus R, Richtmyer R (1977) Similarity solutions for converging shocks. Tech. Rep. LA-6823-MS, Los Alamos Scientific Laboratory

Lazarus RB (1981) Self-similar solutions for converging shocks and collapsing cavities. SIAM Journal on Numerical Analysis 18(2):316-371

Liverts M, Apazidis N (2016) Limiting temperatures of spherical shock wave implosion. Physical Review Letters 116(1):014,501

Luo X, Zhang F, Ding J, Si T, Yang J, Zhai Z, Wen Cy (2018) Long-term effect of Rayleigh-Taylor stabilization on converging Richtmyer-Meshkov instability. Journal of Fluid Mechanics 849:231-244

Luo X, Li M, Ding J, Zhai Z, Si T (2019) Nonlinear behaviour of convergent Richtmyer-Meshkov instability. Journal of Fluid Mechanics 877:130-141

Moutte A (2018) Etude de jets turbulents à masse volumique variable: impact de la variation de masse volumique sur la structure fine et le mélange. PhD thesis, Ecole Centrale de Marseille

Perry RW, Kantrowitz A (1951) The production and stability of converging shock waves. Journal of Applied Physics 22(7):878-886
Ramsey SD, Kamm JR, Bolstad JH (2012) The Guderley problem revisited. International Journal of Computational Fluid Dynamics 26(2):79-99

Russell DA (1967) Shock-wave strengthening by area convergence. Journal of Fluid Mechanics 27(2):305-314

Sembian S, Liverts M (2020) On using converging shock waves for pressure amplification in shock tubes. Metrologia 57(3):035,008

Setchell RE, Storm E, Sturtevant B (1972) An investigation of shock strengthening in a conical convergent channel. Journal of Fluid Mechanics 56(3):505-522

Takayama K, Kleine H, Grönig H (1987) An experimental investigation of the stability of converging cylindrical shock waves in air. Experiments in Fluids 5(5):315-322

Tropea C (2011) Optical particle characterization in flows. Annual Review of Fluid Mechanics 43:399-426

Vandenboomgaerde M, Aymard C (2011) Analytical theory for planar shock focusing through perfect gas lens and shock tube experiment designs. Physics of Fluids 23(1):016,101

Vandenboomgaerde M, Rouzier P, Souffland D, Biamino L, Jourdan G, Houas L, Mariani C (2018) Nonlinear growth of the converging Richtmyer-Meshkov instability in a conventional shock tube. Physical Review Fluids 3:014,001

Whitham G (1958) On the propagation of shock waves through regions of non-uniform area or flow. Journal of Fluid Mechanics 4(4):337-360

Whitham GB (1974) Linear and nonlinear waves, vol 42. John Wiley \& Sons

Williams OJ, Nguyen T, Schreyer AM, Smits AJ (2015) Particle response analysis for particle image velocimetry in supersonic flows. Physics of Fluids 27(7):076,101

Zhai Z, Liu C, Qin F, Yang J, Luo X (2010) Generation of cylindrical converging shock waves based on shock dynamics theory. Physics of Fluids 22(4):041,701 